Recherches en didactique des langues et des cultures

Les cahiers de l'Acedle

\title{
Nissen, E. (2019). Formation hybride en langues : Articuler présentiel et distanciel. Paris : Didier.
}

\section{Grégory Miras}

\section{(2) OpenEdition}

\section{Journals}

Édition électronique

URL : https://journals.openedition.org/rdlc/7867

DOI : $10.4000 /$ rdlc. 7867

ISSN : 1958-5772

Éditeur

ACEDLE

Référence électronique

Grégory Miras, «Nissen, E. (2019). Formation hybride en langues : Articuler présentiel et distanciel. Paris : Didier. », Recherches en didactique des langues et des cultures [En ligne], 17-2 | 2020, mis en ligne le 27 avril 2020, consulté le 17 octobre 2022. URL : http://journals.openedition.org/rdlc/7867 ; DOI : https://doi.org/10.4000/rdlc.7867

Ce document a été généré automatiquement le 17 octobre 2022.

\section{c) (ㄱ)}

Creative Commons - Attribution - Pas d'Utilisation Commerciale - Pas de Modification 4.0 International - CC BY-NC-ND 4.0

https://creativecommons.org/licenses/by-nc-nd/4.0/ 


\title{
Nissen, E. (2019). Formation hybride en langues : Articuler présentiel et distanciel. Paris : Didier.
}

\author{
Grégory Miras
}

1 La monographie Formation hybride en langues: Articuler présentiel et distanciel d'Elke Nissen propose, en 284 pages et 6 chapitres, un panorama large des travaux sur les formations hybrides en langues tout en faisant la synthèse de multiples dispositifs existants. C'est en cela un ouvrage utile pour tous les (futurs) enseignants en didactique des langues, mais aussi les ingénieurs pédagogiques qui souhaiteraient mettre en place ou accompagner la création de formations hybrides en langues. Le titre révèle le choix de l'autrice de retenir, à dessein, l'étiquette de "formation hybride» plutôt que d'autres termes comme le blended learning ou les formations mixtes dont elle considère comme ayant une acception trop large (différentes modalités, approches pédaogiques, etc.). Elle rappelle également la spécificité des formations en langues dans ce type de dispositif. Elke Nissen introduit ou conclut les chapitres par un ou plusieurs encadrés synthétiques, et guide ainsi le lecteur pas à pas vers les points importants à retenir. Cet accompagnement réussi du lecteur est complété par des exemples et des modélisations schématiques de dispositifs.

2 Le chapitre 1 intitulé «Cerner la formation hybride en langues (FHL) » souligne avec pertinence la dimension d'ingénierie pédagogique de cet ouvrage et pose son cadre général. En effet, il débute par la présentation de trois exemples de FHL qui permettent au lecteur de mieux cerner, à la fois les contours, mais aussi les variations possibles en termes de modélisations de dispositifs hybrides. Ces dernières sont le résultat des possibilités multiples, en termes d'outils mobilisés, et de transformation du dispositif lui-même en fonction de ses acteurs (objectifs, besoins, possibilités). Ces possibilités sont d'autant plus importantes que l'articulation entre présence et distance permet des variations infinies. Face à cette multitude de voies ouvertes, l'autrice rappelle son choix de se focaliser sur une analyse micro, voire méso, des FHL (p. 33). Ainsi, une première 
définition générale est proposée pour ce terme permettant au lecteur de mieux comprendre les frontières de ce que l'autrice considère comme les FHL.

La formation hybride en langues (FHL) articule au sein de son ou de ses scénarios(s) pédagogique(s) deux modes, le distanciel et le présentiel. Elle correspond à une forme de formation spécifique, dont chacun des modes est également spécifique par rapport à une formation qui se déroulerait entièrement, respectivement, à distance ou en présentiel. La FHL fait appel à un accompagnement et elle intègre l'interaction, dans un mode comme dans l'autre, à travers la présence d'un (ou plusieurs) scénario(s) de communication. Elle se base, au moins partiellement, sur une pédagogie active. (Nissen, $2019: 35$ ).

Il s'agit donc d'une coprésence humaine (des interactions physiques ou à distance), reprenant les principes du socioconstructivisme, qui présente des particularités tant en termes de modalités d'organisation (un accompagnement), de moyens d'action (présence ou distance) et de dispositions pédagogiques (scénario, pédagogie active). Cependant, pour respecter les principes de l'hybridité, il est nécessaire qu'une phase de non-coprésence physique soit mise en place à un moment donné du dispositif. Cette phase doit nécessairement être incluse (et accompagnée) dans le scénario global pédagogique. L'autrice souligne que la FHL est une formation spécifique qui nécessite de déconstruire un agir professoral établi, mais aussi le distanciel pur, en insistant sur l'articulation structurelle avec les moments en présence. Cette articulation est à la fois pensée par l'enseignant et par les apprenants qui disposent de clés pour la construire. Cette construction prend la forme d'une scénarisation (avec des granularités multiples) dont l'objectif est une structuration cohérente de la formation. Dans le cas de la didactique des langues, un scénario de communication - l'explicitation de la manière dont les acteurs vont communiquer entre eux, sera nécessaire en admettant un ensemble de possibilités (plutôt que de contraintes). L'autrice rappelle qu'en matière de FHL contrairement aux situations d'autoapprentissage, qui conduisent à un fort taux d'abandon, il y a toujours la présence d'un enseignant qui pourra être concepteur, tuteur ou les deux.

4 A partir de ce cadre général, Elke Nissen propose d'«identifier les paramètres de l'articulation du distanciel et du présentiel» (p. 68) ce qui constitue le corps du chapitre 2. L'autrice propose trois points nécessaires pour la réussite des FHL. En premier lieu, le «fil rouge", que l'autrice définit comme la colonne vertébrale du scénario pédagogique, assure la cohérence du dispositif dans son intégralité tout en intégrant deux aspects : une unité d'ensemble (une méthodologie, un aspect langagier ou une compétence) et le scénario de communication (petits et grands groupes tutorés en présentiel ou en télécollaboration (a)synchrones). Le deuxième point dépend du premier et il correspond à l'alternance fonctionnelle entre les deux modes en ce qui concerne les habiletés langagières. En effet, chaque mode présentera une fonction particulière tout en étant complémentaire de l'autre. Par exemple, le présentiel permet des interactions verbales synchrones sans outil numérique tiers et le distanciel rend possible les interactions pour des personnes dans des espaces géographiques éloignés. Il s'agit là de voir comment ces deux modes se complètent (même si chacun a ses spécificités) afin de maintenir le «fil rouge». Cette alternance permet notamment d'accorder aux apprenants une liberté et une flexibilité plus grandes parce que le distanciel permet à chaque apprenant de trouver son propre rythme et ainsi de disposer d'une certaine individualisation. La logique de cette alternance se retrouve dans la question de l'évaluation, qui peut se faire en miroir en présence et à distance, et porter sur des éléments vus dans un mode comme dans l'autre. Au-delà des enjeux de 
l'évaluation valables pour toute formation, l'autrice rappelle qu'il est nécessaire que le travail effectué dans ces deux modes soit évalué (de manière formative ou sommative) pour ne pas donner l'impression que l'un ou l'autre est moins important ce qui pourrait provoquer un désinvestissement des apprenants. Elke Nissen donne plusieurs pistes sur les moyens de maintenir l'investissement: chaque tâche est évaluée, l'apprenant comprend le rôle d'une activité au sein des objectifs de la formation, le résultat d'une tâche s'imbrique dans la tâche suivante, un journal de bord permet de rendre compte des activités de l'apprenant. Le facteur temporel est le dernier point de cette dimension, il se reflète dans la structuration du scénario avant, pendant et après, de manière régulière ou plus asymétrique. Au sein de cette deuxième dimension, tournée autour de l'alternance et de la complémentarité, l'autrice rappelle néanmoins qu'il existe presque toujours un «mode pilier» (lead mode) - la dominance d'un mode sur l'autre, ce qui est aussi le cœur de la réussite des FHL. Ce mode pilier est déterminé en prenant 4 caractéristiques: le mode dans lequel (1) les consignes sont données, (2) le déroulement de la formation est expliqué, (3) les apprenants passent le plus de temps et (4) sa conception a été pensée. Il ne s'agit là pas d'une prédétermination (ce mode peut changer au cours du dispositif) ni d'une domination (il y a alternance) mais c'est ce qui permet d'afficher clairement le fonctionnement de la formation aux apprenants. Pour finir, le troisième point pose la question du choix des outils et notamment celui des critères de leur sélection (accessibles, adaptés, au service des objectifs pédagogiques).

5 Suite à cette proposition d'articulation entre présentiel et distanciel, le chapitre 3 montre l'intérêt de «s'appuyer sur une approche par tâche(s) ». L'autrice rappelle que la notion de "tâche " est ancienne, même si les auteurs cités ne le mentionnaient pas de cette manière (Dewey, Freinet, etc.), et si cette étiquette a pris une ampleur particulière avec l'avènement de l'approche actionnelle. Il s'agit avant tout de reconnaitre l'action comme vecteur de sens pour l'apprentissage en lui donnant un but ancré dans les pratiques de la société comme élaborer un produit, savourer une expérience esthétique, résoudre un problème ou atteindre un certain degré de compétence. Les compétences visées ne sont pas forcément langagières et s'appuient sur un contexte social entourant l'apprenant ou son futur. A partir de ce positionnement général, plusieurs notions sont explicitées : la tâche complexe, qui comporte des sous-tâches (contrairement à une tâche simple), et qui n'est pas forcément finale dans le dispositif; les sous-tâches ou étapes sont orientées vers l'accomplissement de la tâche; les activités d'entrainement qui visent à préparer des compétences utiles pour la réalisation de la tâche; et enfin, des aides qui facilitent le processus de réussite. Dans ce cadre, trois types de scénarios pédagogiques se détachent :

- Une seule tâche comme élément structurant : l'objectif est la réalisation d'une tâche unique qui se décompose en différentes étapes prenant la forme d'unités ou de modules successifs.

- Plusieurs tâches s'enchainent et forment ensemble la colonne vertébrale du scénario pédagogique : le scénario pédagogique se compose de plusieurs tâches qui se suivent d'après une logique variable. Cette logique peut s'appuyer sur la nature des tâches, leur recoupement partiel ou une situation globale partagée.

- Le même type de tâche est proposé de manière itérative (répétée et à intervalle régulier).

6 De manière transversale, la notion de tâche questionne les critères de son authenticité (sociale). Pour l'autrice, elle peut prendre quatre formes : (1) la préparation d'une tâche sociale réelle, (2) une tâche s'inscrivant dans un objectif éducationnel, (3) une 
simulation soit une tâche "sociale " mais reproduite dans le cadre pédagogique, ou bien (4) les objectifs d'apprentissage et sociaux sont combinés. Elke Nissen propose de questionner l'authenticité de la tâche du point de vue des acteurs : l'enseignant et/ou le concepteur mais aussi les apprenants, voire le public. Elle rappelle que cette authenticité est construite et qu'elle peut reposer sur la "sincérité » de l'apprenant dans son investissement dans la tâche, sa plausibilité, les enjeux ou sa signification notamment pour l'apprenant ou encore une adhésion motivée de ce dernier. L'importance du contrat social, notamment avec le public, est un facteur déterminant dans l'investissement des apprenants.

7 Le chapitre 4 développe les modalités de combinaison entre télécollaboration et présentiel. La télécollaboration est définie par Elke Nissen comme une interaction sociale, en ligne, médiatisée par le langage entre des apprenants éloignés géographiquement, avec un objectif d'apprentissage langagier, interculturel, technique et/ou disciplinaire. Elle se déroule dans un cadre institutionnel qui intègre au moins deux partenaires incluant des enseignants ou des tuteurs qui proposent des tâches collectives. C'est un bon moyen pour les apprenants de pratiquer une langue et elle peut être perçue (voire reconnue de plus en plus) comme une mobilité virtuelle. Plusieurs éléments permettent de distinguer les différentes formes de télécollaboration: les objectifs visés, la configuration langagière, la configuration de participation, la temporalité et les outils, la durée et la fréquence ou encore l'intégration dans le cursus. L'une des limites de ce type de dispositif est le caractère chronophage pour les enseignants et, potentiellement, pour les apprenants. Une fois ces éléments posés, l'autrice commente plusieurs exemples de FHL collaborative dans des contextes variés en utilisant des outils différents et portant sur des langues variées (anglais, langues romanes, etc.). Ainsi, il est proposé que les interactions au sein de FHL disposent de propriétés spécifiques et qu'elles nécessitent donc un séquençage particulier. Le premier cas repose sur un enchaînement d'étapes d'une tâche-projet (par exemple: briser la glace, échange d'opinions, préparation du projet final, rédaction/publication du projet final). Le deuxième cas reprend le séquencement fondé sur Willis (1996) et correspond à un enchainement d'activités pédagogiques qui visent la réussite du projet collaboratif, se rapprochant de savoir-faire (par exemple : établir le contact, accomplissement d'activité, phase analytique/réflexive). Le troisième et dernier cas, l'activité itérative (répétée) est souvent à distance et elle permet d'anticiper chaque séance de télécollaboration (par exemple, lire et travailler des documents audio avant d'en discuter avec ses pairs). Dans tous les cas, la collaboration humaine permet une authenticité des échanges qui augmente l'investissement des apprenants. Cependant, elle nécessite que ces derniers prennent des risques ce qui n'est pas évident pour tous les individus. Pour terminer, il est question des spécificités dans l'articulation des modes de la FHL collaborative. La pluralité des scénarios de communication est le reflet de la variété des situations de chaque partenaire impliqué. Il en va de même pour l'enseignant qui pourra adopter différents rôles, mais celui de régulateur sera d'autant plus important qu'il vérifiera le bon déroulement des interactions nécessaires pour la réalisation des objectifs. La particularité des FHL collaboratives repose sur le fait qu'il existe deux piliers : le présentiel car il est le mode référent localement pour chaque partenaire (présentation du scénario, discussions guidées, négociations, etc.) et le distanciel puisque le scénario pédagogique (les échanges collaboratifs) est centré sur ce mode. 
8 Pour terminer, Elke Nissen s'intéresse, dans un cinquième chapitre, aux modalités d'accompagnement de l'apprenant dans une FHL. Tout d'abord, elle porte son attention sur l'autonomisation de l'apprenant qu'elle définit comme une compétence qui permet d'être acteur de sa formation et de la réussir, et qui s'acquiert. Il s'agit de soutenir l'apprenant pour qu'il soit capable de réussir les activités pédagogiques et qu'il sache gérer différents degrés de responsabilité (organisationnelle, méthodologique, sociale, etc.). Ce soutien passe notamment par la structuration du scénario pédagogique, qui est clair dès le début de la formation, tout en laissant aux apprenants une certaine liberté notamment dans le choix des outils et des ressources. Plusieurs exemples sont ainsi donnés pour illustrer les différents types de soutien. Cependant, elle reconnait que ce type les FHL ne convient pas à tous les apprenants pour des raisons diverses : maitrise des outils informatiques, changement dans les habitudes d'apprentissage, faible engagement, etc. De ce fait, la présence sociale de l'enseignant-tuteur est déterminante car elle contribue à l'engagement de l'apprenant et donc à la réussite de l'hybridation. Toutefois, certaines des fonctions de l'enseignant-tuteur sont également prises en charge par l'environnement techno-pédagogique. Ainsi, l'enseignant-tuteur a des rôles multiples qui évoluent au cours des scénarios pédagogique et de communication (aide pour gérer et planifier l'apprentissage, création d'un climat de travail favorable, indication des critères d'évaluation, etc.). Dans les particularités des FHL, notons que lorsque des enseignants et/ou tuteurs reprennent une formation sans en avoir pris en charge une partie de la conception (et donc pensé le fil rouge), les principaux enjeux sont de saisir comment fonctionne cette formation afin d'être capables de s'approprier l'approche méthodologique pour mieux expliquer le scénario pédagogique aux apprenants. D'autres facteurs professionnels sont également centraux : pouvoir faire évoluer le scénario pédagogique, intégrer une équipe de tuteurs, se sentir à l'aise avec les outils ou apprécier les contenus de la formation. La formation des enseignants au scénario pédagogique est indispensable. Dans les FHL, l'accompagnement par les pairs est tout aussi important que celui apporté par l'enseignant et l'environnement technopédagogique. Dans une perspective socioconstructiviste, Elke Nissen rappelle la nécessité des interactions humaines dans le développement langagier en tant que production sociale. Cependant, si ces interactions sont fréquentes dans les FHL, le travail collectif y est parfois erratique. Pourtant, favoriser la création de communautés d'apprenants dans les deux modes (présentiel et distanciel) est crucial surtout si l'on adopte une pédagogie centrée sur l'action et les apprenants (par exemple, la résolution de problèmes). Pour l'autrice, le travail en groupe est un élément facilitant l'autonomisation individuelle. Les principaux enjeux autour de l'intégration du travail en groupe en FHL sont la nécessité d'une plus grande autonomie organisationnelle des apprenants, la possibilité d'avoir accès à des outils robustes permettant le travail en groupe à distance, la reconnaissance du travail que cela implique pour l'enseignant en dehors du présentiel, et la nécessité d'un panachage d'évaluations individuelles et collectives. Il devient alors nécessaire de distinguer la collaboration (les membres du groupe négocient conjointement l'ensemble des étapes de production) et la coopération (les membres du groupe se divisent le travail puis assemblent à la fin ces parties qui forme une production conjointe). Favoriser une démarche de collaboration implique la création d'une communauté, et donc d'un sentiment d'appartenance au groupe, qui participera au succès de la FHL.

9 Le chapitre 6 est en fait la conclusion de l'ouvrage. Il propose des outils tangibles pour les acteurs de la formation en langue: une taxonomie synthétisant les différents 
paramètres qui interviennent dans l'articulation des modes présentiel et distanciel, mais aussi une typologie des FHL et des conditions de leur mise en place dans d'autres contextes que celui de l'enseignement supérieur.

Dans son ensemble, cet ouvrage répond au cadre posé par l'autrice, à savoir, fournir aux acteurs de l'enseignement-apprentissage des langues un outil pratique qui organise, dans une perspective d'ingénierie pédagogique et de formation, les clés pour la mise en place de formations hybrides en langues. L'organisation même de l'ouvrage permet à son lecteur de trouver des éléments de synthèse (des encadrés, des taxonomies, des schématisations) mettant en évidence les caractéristiques de FHL complexes mais aussi des descriptions détaillées qui fournissent le matériel nécessaire pour construire des scénarios pédagogiques et de communication opérationnels. Le fonctionnement de la réflexion en entonnoir (partir d'exemples variés de FHL pour déterminer des clés de réussite) fait son originalité et sa force pour les acteurs de la didactique des langues-cultures. Cet ouvrage joue un rôle important pour souligner le fait que les formations hybrides en langues sont un "genre de formation spécifique " (p. 17) et doivent être conçues dès le début comme telles en dehors de toute volonté de transposer des approches du présentiel vers l'hybride. Ce point, qui pourrait sembler négligeable, est nécessaire pour inciter les institutions à mieux reconnaitre ce type de formations alors qu'elles fonctionnent souvent administrativement sur la base d'un cours magistral. Ces difficultés sont cependant partagées avec d'autres approches que les FHL lorsqu'il est nécessaire de déconstruire les espaces physiques et temporels de la « salle de classe ».

11 Ce positionnement général en ingénierie pédagogique de la question des FHL amène à une focalisation «de niveau micro ou encore méso » (p. 16). Tout en reconnaissant qu'une démarche globale sur l'articulation des différents niveaux (micro, méso et macro) n'est pas le centre de l'ouvrage et nécessite d'intégrer les travaux en histoire de la didactique tout autant qu'en politique éducative, une telle démarche permettrait néanmoins de mieux situer ces pratiques dans une démarche d'analyse didactique glocale (Robertson, 1995) et de circulation des représentations. Il nous semble qu'un certain nombre d'éléments pourraient être pris en compte en complément de cet ouvrage pour aller plus loin dans une démarche holistique. Tout d'abord, il nous semble important de remarquer, tout comme le fait l'autrice dans la partie 6.3, «Conditions pour la mise en place d'une FHL dans d'autres contextes que celui de l'enseignement supérieur» (p. 254), que la majorité des exemples de dispositifs exploités a été mise en place à l'université. Or, d'une part, les enjeux mis en évidence dans les FHL posent l'importance de l'implémentation de ces dispositifs dès le système primaire et secondaire pour réduire les conséquences inhérentes à des approches dominantes actuelles transmissives et fermées dans la "salle de classe " (notamment le niveau réel en langues des bacheliers). Mais aussi, et d'autre part, il semble nécessaire de préciser la réalité des pratiques sur le terrain de l'enseignement des langues. Si la recherche montre clairement l'apport des formations hybrides par rapport au tout présentiel ou à l'autoformation (Narcy-Combes et al., 2019) et si les enseignants peuvent être (de plus en plus) convaincus par ce type de dispositifs, il n'en reste pas moins vrai que de nombreuses limites expliquent le faible taux de mise en place de FHL dans les institutions. Ces limites sont, entre autres, la non-reconnaissance du temps de travail supplémentaire que cela nécessite pour les enseignants de langues, la modification hebdomadaire des cohortes d'apprenants et la non-rémunération de la formation continue des enseignants. Ainsi, si l'on reconnait qu'il n'existe pas de «résistance au 
changement » (Duclos, 2015) de la part des enseignants face au numérique, ce sont bien les conditions d'exercice du métier d'enseignant qui ne permettent pas toujours de penser au mieux et de mettre en place ce type de formations. Toutefois et dans le même temps, on constate l'augmentation de recrutement d'ingénieurs pédagogiques (souvent en lien avec le numérique), dans les structures qui, comme les universités, le peuvent, pour faciliter et dynamiser le montage de dispositifs de ce type et viser la formation pédagogique des maitres de conférences stagiaires (Décret n 2017-854 du 9 mai 2017). Cet accroissement participe à la pression perçue par les institutions pour se présenter comme « innovantes » (MESRI, 2018) parfois au détriment d'une réflexion pédagogique sur les besoins réels du public ciblé. Cette réalité professionnelle est également au centre de la question posée par l'autrice sur le rôle des tuteurs de $2^{\mathrm{e}}$ génération (p. 225). En effet, avec la démocratisation des formations hybrides ou totalement à distance, le métier de «tuteur » est de plus en plus reconnu et intégré dans des fiches métier des établissements. Toutefois, la rémunération dépend largement des conventions de l'institution (équivalence TD, rémunération à la tâche) et catalyse la question de la précarisation des métiers de l'enseignement (le contrat de base à l'université étant la vacation ce qui implique d'avoir un emploi principal). Or, la légitimation de ces nouveaux rôles, sur le plan professionnel, est un levier nécessaire si l'on veut la réussite à long terme de ces dispositifs hybrides (Burrows \& Miras, 2019).

Un deuxième point, qui nous semble soulevé par ce type de travail de synthèse, en miroir à l'articulation des deux modes proposée par Elke Nissen, est lié à l'articulation entre les pratiques de terrain et les démarches de théorisation. Si l'autrice indique clairement tout au long de l'ouvrage, son inscription dans une approche socioconstructiviste de l'apprentissage (Vygotsky) et dans des pédagogies centrées sur l'action (Dewey, Brunet), ces apports scientifiques (complétés par d'autres) auraient pu permette une remise en question de l'impact du CECRL et de l'approche actionnelle sur la conception des curricula - omniprésents dans les dispositifs retenus qui répondent à des demandes institutionnelles. En effet, si le CECRL n'est pas un outil issu de la recherche scientifique, Macaire et al. (2010:15) rappellent néanmoins qu'il a acquis une "autorité incontestable» notamment pour les acteurs de terrain. Cette autorité construite pose la question de l'impact de ce type de document sur les choix retenus (pédagogiques et didactiques) lors de la construction de formations hybrides. Elle peut également être source de perturbation lorsqu'il est question de définir des notions complexes comme celle de "tâche ", ou de réflexions dites interculturelles, notamment dans le cas mentionné par l'autrice où le scénario de communication n'est pas déterminé par une méthodologie d'enseignement-apprentissage mais directement par une compétence ou un aspect langagier. Or, il parait nécessaire de rappeler que toute liberté pédagogique est conditionnée à une responsabilité épistémologique (la capacité à se situer scientifiquement par rapport à une approche didactique) ce qui implique que tout choix (éventuellement revu à postériori) de conception pédagogique repose sur des éléments issus de la recherche scientifique. Pour terminer, certaines notions polysémiques et polyréférentielles, comme celle d'autonomie (p. 205), traitées au regard d'autres disciplines, comme la psychologie ou les neurosciences, nous amènent à en concevoir les limites. Ainsi, l'autonomie, vue comme une forme d'agentivité, pose la question de la marge de manœuvre des individus à l'accepter ou la refuser dans un dispositif centré sur l'apprenant et l'action. Cette problématique est d'autant plus importante que la recherche montre que ce type de pédagogie active peut être facteur d'anxiété pour l'individu-apprenant (Arnold, 2000). 
L'ouvrage d'Elke Nissen est un support qui apporte de nombreuses réponses à des acteurs de la didactique des langues qui se trouvent confrontés à la mise en place de formations hybrides en langues ou à une envie de les tester. C'est un ouvrage qui prend logiquement sa place dans une collection (Langues \& didactiques aux Editions Didier). En effet, un certain nombre des questions qu'il soulève sur la complémentarité entre théories et pratiques tout en faisant appel à de nombreuses disciplines, tant il s'agit d'objets complexes, ont eu des réponses dans l'ouvrage qui le précède dans la collection (Narcy-Combes \& Narcy-Combes, 2019) et dont la conclusion souligne que les formations hybrides semblent les plus pertinentes pour répondre aux problèmes que soulève aujourd'hui l'apprentissage des langues en milieu institutionnel.

\section{BIBLIOGRAPHIE}

Arnold, J. (2000). Affect in Language Learning. Cambridge: Cambridge University Press.

Burrows, A. \& Miras, G. (2019). Pratiques numériques en langues : de la verbalisation à l'analyse des préoccupations enseignantes. Alsic [En ligne], vol. 22. http://journals.openedition.org/alsic/ 3549 (Consulté le 14/10/19).

Duclos, A.-M. (2015). La résistance au changement : un concept désuet et inapproprié en éducation. Psychologie \& Éducation, $\mathrm{n}^{\circ} 1$. p. 33-45.

Macaire, C., Narcy-Combes, J.-P. \& Portine, H. (2010). Interrogations épistémologiques en didactique des langues. Le français dans le monde, Recherches et applications. Paris : CLE International.

MESRI (2018). Les innovations pédagogiques numériques et la transformation des établissements d'enseignement supérieur. Rapport $\mathrm{N}^{\circ}$ 2018-049, juin. https://cache.media.enseignementsuprecherche.gouv.fr/file/2018/43/6/IGAENR-Rapport-2018-049-Innovations-pedagogiquesnumeriques-transformation-etablissements-enseignement-superieur-2_980436.pdf (Consulté le 10/10/19).

Narcy-Combes J.-P., Narcy-Combes M.-F. (2019). Cognition et personnalité dans l'apprentissage des langues. Relier théorie et pratique. Paris : Didier.

Narcy-Combes, M.-F., Narcy-Combes, J.-P., MacAllister, J., Leclerc M. \& Miras, G. (2019). Language Learning and Teaching in a Multilingual World. Bristol: Multilingual Matters.

Robertson, R., (1995), Glocalization: Time-Space and Homogeneity-Heterogeneity. Dans Featherstone, M., Lash, S. \& Robertson, R. (dir.), Global Modernities. Londres : Sage Publications, p. 25-44.

\section{INDEX}

Thèmes : Comptes rendus 
AUTEUR

\section{GRÉGORY MIRAS}

Université de Rouen Normandie, DYLIS-EA7474

Grégory Miras est maître de conférences au laboratoire DYLIS (EA7474) à l'Université de Rouen Normandie. Ses thématiques de recherche portent à la fois sur la compréhension des mécanismes de perception/production des sons en langue étrangère mais aussi sur les technologies numériques pour l'enseignement/apprentissage des langues. Il s'intéresse également à l'épistémologie de la connaissance didactique.

gregory.miras[at]univ-rouen.fr

https://sites.google.com/site/gregorymiras/home 